\title{
Katarzyna Chlewicka
}

\section{Schwieriger Kulturtransfer}

\author{
Lorenz Christoph Mizler de Kolofs Bemühungen um die \\ Etablierung gelehrter Zeitschriften in Polen am Beispiel der \\ Warschauer Bibliothek (1753-55)
}

Zusammenfassung: Mit der Gründung der Warschauer Bibliothek, der ersten gelehrten Zeitschrift in Warschau, setzte sich der aus Sachsen stammende Lorenz Christoph Mizler de Kolof (1711-1778) für den Transfer aufklärerischer Wissens- und Bildungspraktiken in Polen ein. Die Herausgabe der Zeitschrift wurde jedoch von Konflikten und Spannungen begleitet und musste relativ schnell aufgegeben werden. Die Warschauer Bibliothek stellte in der polnischen Öffentlichkeit eine fremdartige Erscheinung in zweifacher Hinsicht dar: sowohl als ein Presseorgan, ein gelehrtes Journal an sich und damit eine dem polnischen Kulturleben bisher wenig vertraute öffentliche Institution, als auch im Sinne einer kritischen Herangehensweise an das Wissen und Denken, die diese Institution forderte. Die Gründung der Warschauer Bibliothek führte somit nicht automatisch zu einem Aneignungsprozess aufklärerischer Denkmuster und Praktiken der Wissensvermittlung.

Schlagwörter: Warschauer Bibliothek; Lorenz Christoph Mizler de Kolof; gelehrte Zeitschriften; Kulturtransfer; Aufklärung

Abstract: The Warschauer Bibliothek, the first scholarly journal in Warsaw, was founded by Lorenz Christoph Mizler de Kolof (1711-1778) - who originally hailed from Saxony. However its publication, which advocated the transfer of enlightened knowledge and education practices in Poland, resulted in a number of conflicts and tensions that led to it being quickly abandoned. The Warschauer Bibliothek was an unfamiliar medium for the Polish public in two distinct ways: firstly as a publication in itself, scholarly journals were not well-known phenomena in Polish society at that point; and secondly that Poles would be unfamiliar with the journal's critical approach to knowledge and thinking. As a result, the formation of the Warschauer Bibliothek did not automatically lead to an adoption of enlightened ways of thinking and practices of transferring knowledge.

Keywords: Warschauer Bibliothek; Lorenz Christoph Mizler de Kolof; scholarly journals; cultural transfer; Enlightenment

Katarzyna Chlewicka, Dr., Uniwersytet Mikotaja Kopernika w Toruniu, Katedra Literatury, Kultury i Komparatystyki Niemieckiego Obszaru Językowego, ul. W. Bojarskiego 1, 87-100 Toruń, Polen, kch@umk.pl 


\section{Einleitung}

Lorenz Christoph Mizler de Kolof (1711-1778) hat sich einmal selbst in einem seiner Zeitschriftenbeiträge von 1754 als einen „von den ersten Aposteln der Wolfischen Philosophie“ in Polen charakterisiert. ${ }^{1}$ An diese Selbsteinschätzung knüpfte dann im 20. Jahrhundert die polnische Forschung an, als sie Mizler de Kolof als „Boten der deutschen Aufklärung“ oder gar „Wegbereiter der Aufklärung“ in Polen bezeichnete und ihm damit einen besonderen Status in der polnischen Literaturgeschichtsschreibung einräumte. ${ }^{2}$ Und tatsächlich würde es schwerfallen, im Polen des 18. Jahrhunderts einen anderen ,Zuwanderer‘ aus dem europäischen Ausland zu finden, der sich für den Transfer der aufklärerischen Wissens- und Bildungspraktiken in so unterschiedlichen Bereichen und auf eine so intensive Weise einsetzte, wie er es tat.

Der aus Sachsen stammende (wenn auch aus Heidenheim in Mittelfranken gebürtige) Mizler de Kolof (die polnische Variante seines Namens lautet Wawrzyniec Krzysztof Mizler de Kolof) kam im Jahre 1743 in die polnische Adelsrepublik, nach einem mehrjährigen Aufenthalt in Leipzig, wo er Philosophie, Theologie, Musikwissenschaft und Medizin studiert hatte und dabei zum eifrigen Anhänger Christian Wolffs geworden war. Die ersten Jahre in Polen verbrachte er in der Ortschaft Końskie, auf dem Gut des einflussreichen Magnaten und späteren Krongroßkanzlers Jan Małachowski, der ihm auf Vermittlung Johann Christoph Gottscheds die Stelle des Hofmeisters angeboten hatte. Im Jahre 1749, als ihm die ersehnte Professur an der Leipziger Universität verwehrt blieb, ließ er sich in Warschau nieder, wo er nachfolgend fast 30 Jahre lang als Historiograph, Herausgeber, Übersetzer, Buchdrucker und Mediziner tätig war. ${ }^{3}$ Die lange Liste der Pionierleistungen Mizler de Kolofs in Polen

1 Warschauer Bibliothek oder gründliche Nachrichten nebst unpartheyischen Urtheilen von allen Büchern und Schrifften, sowohl alten als neuen, so iemals in Pohlen herausgekommen, oder von auswärtigen Gelehrten in Pohlen geschrieben worden. Worinnen zugleich von dem dermaligen Zustand der Gelehrsamkeit in Pohlen zuverläßige Nachricht gegeben wird, sammt den Lebensläufen der polnischen Mäcenaten und merkwürdigen Gelehrten. 2. Teil (1754), S. 170. Ein Digitalisat der Zeitschrift ist über den Katalog der Bayerischen Staatsbibliothek einsehbar unter: https://opacplus.bsb-muenchen. de/title/BV037432446 (06.02.2020).

2 Mieczysław Klimowicz: Polsko-niemieckie pogranicza literackie w XVIII wieku. Problemy uczestnictwa w dwóch kulturach [Polnisch-deutsche literarische Grenzgebiete im 18. Jh. Probleme der Teilhabe an zwei Kulturen]. Wrocław-Warszawa-Kraków 1998, S. 17; ders.: Mizler de Kolof. Redaktor i wydawca [Mizler de Kolof. Redakteur und Herausgeber]. In: Ders., Roman Kaleta (Hg.): Prekursorzy Oświecenia [Wegbereiter der Aufklärung]. Wrocław 1953, S. 220 - 333, hier S. 220.

3 Vgl. Lutz Felbick: Lorenz Christoph Mizler de Kolof. Schüler Bachs und pythagoreischer „Apostel der Wolffischen Philosophie“. Hildesheim-Zürich-New York 2012, S. 358-417; Franz Wöhlke: Lorenz Christoph Mizler. Ein Beitrag zur musikalischen Gelehrtengeschichte des 18. Jahrhunderts. Würzburg 1940, S. 3-37. 
umfasst unter anderem kritische Editionen polnischer Geschichtsquellen, ${ }^{4}$ Übersetzungsarbeiten, ${ }^{5}$ aber auch seine Briefe über das Warschauer Theater, die erste theoretische Abhandlung über das bürgerliche Theater in Polen. ${ }^{6}$ Doch die Aktivitäten, mit denen er am intensivsten als ,Bote der Aufklärung' in Polen agierte und sich ganz bewusst für einen weit verstandenen Kulturtransfer einsetzte, waren seine kontinuierlichen Bemühungen um die Etablierung eines Zeitschriftenmarktes in der polnischen Hauptstadt. Von den vier ersten in Warschau im 18. Jahrhundert erschienenen gelehrten Zeitschriften wurden drei als Einzelunternehmen von Mizler de Kolof gegründet (Warschauer Bibliothek 1753-1755, Acta Litteraria Regni Poloniae et Magni Ducatus Lithuaniae 1755-1756 sowie Nowe wiadomości ekonomiczne i uczone [Neue ökonomische und gelehrte Nachrichten] 1758-61, 1764 und 1767). ${ }^{7}$ Im Jahre 1761 hat er zudem die erste moralische Wochenschrift in polnischer Sprache Patriota Polski [Der polnische Patriot] von Thomas Bauch verlegt und mitfinanziert. ${ }^{8}$ Auch an der Herausgabe des wichtigsten und langlebigsten Presseorgans der polnischen Aufklärung, des Warschauer Monitor (1765-1785), war er als Drucker und zeitweise auch Autor der Beiträge maßgeblich beteiligt. ${ }^{9}$

Mizler de Kolofs Versuche, mit gelehrten Zeitschriften in Polen eine wissenschaftliche Infrastruktur zu schaffen, wie sie sich im Zeitalter der Aufklärung in anderen Ländern Europas entfaltete und wie er sie aus Deutschland kannte, wurden jedoch von Spannungen und Konflikten begleitet. Dass die öffentliche Sphäre in Polen im 18. Jahrhundert anders als in Deutschland funktionierte, war mit dafür verant-

4 Für die Herausgabe der fünfbändigen Sammlung von Chroniken und historischen Schriften Historiarum Poloniae et magni ducatus Lithuaniae scriptorum ... collectio magna (1761-1777) wurde er von König Stanisław II. August Poniatowski mit der Merentibus-Medaille ausgezeichnet. Vgl. Krystyna Korotajowa, Joanna Krauze-Karpińska (Hg.): Drukarze dawnej Polski od XV do XVIII wieku [Die Drucker im alten Polen vom 15. bis zum 18. Jahrhundert]. Bd. 3: Mazowsze z Podlasiem [Masowien mit Podlachien]. Warszawa 2001, S. 228.

5 Insbesondere sei die Übersetzung von Gottscheds Abhandlung Erste Gründe der gesammten Weltweißheit ins Polnische im Jahre 1761 erwähnt, die den Polen die Lehren Wolffs in ihrer Muttersprache zugänglich machte und gleichzeitig einen gelungenen Versuch darstellte, eine polnische philosophische Terminologie zu schaffen. Vgl. Mieczysław Klimowicz: Die Frühaufklärung der Jahre 1733-1763 und die Aufklärung der Periode des Königs Stanisław August im Lichte der deutsch-polnischen literarischen Beziehungen. In: Karol Bal, Siegfried Wollgast, Petra Schellenberger (Hg.): Frühaufklärung in Deutschland und Polen. Berlin 1991, S. 163-172, hier S. 164.

6 Vgl. Gerard Koziełek: Lorenz Mitzler de Kolofs „Briefe über das Warschauer Theater“. In: Ders.: Reformen, Revolutionen und Reisen. Deutsche Polenliteratur im 18. und 19. Jahrhundert. Wrocław u. a. 1990, S. 52-65.

7 Vgl. Danuta Hombek: Prasa i czasopisma polskie XVIII wieku w perspektywie bibliologicznej [Polnische Presse und polnische Zeitschriften des 18. Jahrhunderts aus bücherkundlicher Perspektive]. Kraków 2001, S. 58-72. Der vierte Titel, von dem nur ein Heft im Jahre 1754 erschienen ist, war das Journal Literaire de Pologne von Christian Gottlieb Friese. Vgl. Jerzy Łojek: Bibliografia prasy polskiej 1661-1831 [Bibliographie der polnischen Presse 1661-1831]. Warszawa 1965, S. 16.

8 Vgl. Hombek, Prasa i czasopisma (wie Anm. 7), S. 72f.

9 Vgl. Elżbieta Aleksandrowska: Monitor. 1765-1785. Wrocław u.a. 1976, S. LXXIX. 
wortlich, dass die Herausgabe insbesondere der beiden ersten Periodika nur zögernd voranging und relativ schnell, nach äußerst unregelmäßigem Erscheinen aufgegeben werden musste (von der als Zweimonatsschrift konzipierten Warschauer Bibliothek erschienen 1753-1755 nur vier Hefte, die Acta Litteraria brachten es 1756-1763 auf acht Ausgaben). ${ }^{10}$ Kritische Publikationen ohne Einvernehmen mit der adligen Elite waren nicht erwünscht, kritisches Räsonnement kaum zugelassen. Auch ließ sich das Interesse der polnischen Eliten an einer redaktionellen Mitarbeit und dem Aufbau eines gelehrten Netzwerks in Polen kaum wecken. Die von Mizler de Kolof bewirkte Etablierung gelehrter Periodika in Warschau führte somit nicht automatisch zu einem Aneignungsprozess aufklärerischer Denkmuster und Praktiken der Wissensvermittlung. Der Kulturtransfer, den de Kolof als bewusster Mittler zwischen den Kulturen anstrebte, erwies sich als langwierig und schwierig für beide Seiten.

Im folgenden Beitrag soll Lorenz Christoph Mizler de Kolofs möglicherweise wichtigste Initiative in Polen, die Gründung der Warschauer Bibliothek, in den Blick genommen werden, und zwar als Beispiel eines nicht einfachen Kulturtransfers zwischen der deutschen und der polnischen Kultur im Zeitalter der Aufklärung. Die Warschauer Bibliothek, die in die Geschichte eingegangen ist als die erste gelehrte Zeitschrift in Polen (allerdings nicht ganz zu Recht, denn ihr gingen die gelehrten deutschsprachigen Periodika aus den königlich preußischen Städten Thorn (Toruń) und Danzig (Gdańsk) sowie aus dem großpolnischen Lissa (Leszno) voran), ${ }^{11}$ fand im Polen des 18. Jahrhunderts nicht die Resonanz, die sich Mizler de Kolof erhofft hatte. Sie stellte in der polnischen Öffentlichkeit eine fremdartige Erscheinung in zweifacher Hinsicht dar, sowohl als ein Presseorgan, ein gelehrtes Journal an sich und damit eine dem polnischen Kulturleben bisher wenig vertraute öffentliche Institution, als auch im Sinne einer kritischen Herangehensweise an das Wissen und Denken, die diese Institution forderte.

10 Vgl. Hombek, Prasa i czasopisma (wie Anm. 7), S. 62, 348f. Die insgesamt acht auf die Jahre 1755 und 1756 datierten Nummern der Acta Litteraria erschienen tatsächlich unregelmäßig in den Jahren $1756-1763$.

$11 \mathrm{Zu}$ den ersten gelehrten Periodika in Thorn vgl. den Beitrag von Anna Mikołajewska in dieser Themenausgabe. Dass die polnische Forschung die deutschsprachigen Periodika aus den Städten des Königlichen Preußen sowie aus Lissa oft übergangen hat, lag weitgehend an den Publikationen von Jerzy Łojek, insbesondere an seiner 1965 veröffentlichten Bibliographie der polnischen Presse (Bibliografia prasy polskiej 1661-1831 [Bibliographie der polnischen Presse 1661-1831]), die jahrzehntelang in der Presseforschung Kanonstatus genoss, obwohl sie zahlreiche Titel aus Danzig, Thorn, Marienburg (Malbork) und Lissa ausschloss. Inzwischen haben mehrere Forscher die Erweiterung der Perspektive von Łojek gefordert. Vgl. insbesondere Stanisław Salmonowicz: Die Zeitschriftentypen in Polen und ihre Rolle als Förderer der Aufklärung. In: Istvan Fried, Hans Lemberg, Edit RosenstrauchKönigsberg (Hg.): Zeitschriften und Zeitungen des 18. und 19. Jahrhunderts in Mittel- und Osteuropa. Essen 1987, S. 65-90; Danuta Hombek: O potrzebie nowej bibliografii polskich gazet i czasopism XVIII wieku [Über das Desiderat einer neuen Bibliographie der polnischen Zeitungen und Zeitschriften]. In: Rocznik Bibliologiczno-Prasoznawczy 2/13 (2010), S. 13-33. 


\section{Die Entstehung der Warschauer Bibliothek}

Zur Zeit der Ankunft Mizler de Kolofs in Polen (also kurz vor der Mitte des 18. Jahrhunderts) gab es in Warschau so gut wie keinen Pressemarkt. Die einzigen in der polnischen Hauptstadt erscheinenden Periodika waren die von den Orden der Jesuiten und Piaristen verlegten Zeitungen Kurier polski [Der polnische Kurier] und Uprzywilejowane wiadomości $z$ cudzych krajów [Privilegierte Nachrichten aus fremden Ländern] ${ }^{12}$ Davon hob sich stark die Situation in Deutschland ab, wo bereits in den ersten beiden Jahrzehnten des 18. Jahrhunderts das Angebot an gelehrten Periodika so groß war, dass man vom „Ausufern der Journal- und Zeitschriften-Mode“ spricht. ${ }^{13}$ Mizler de Kolof, der noch in Leipzig zwei eigene Zeitschriften, die Musikalische Bibliothek (1736-1754) und den Musikalischen Staarstecher (1739-1740), gegründet hatte, ${ }^{14}$ scheint daher mit seiner Warschauer Bibliothek in Polen tatsächlich Neuland betreten zu haben. Auf den unverkennbaren Unterschied in der Entwicklung des deutschen und des polnischen Pressemarktes im 18. Jahrhundert weist er ausdrücklich in den allerersten Sätzen der Warschauer Bibliothek hin:

Woran Deutschland einen Ueberfluß hat, daran hat Pohlen einen Mangel. Ich meine, wenn ganz Deutschland mit Tagebüchern, Monatsschriften, gelehrten und halbgelehrten Zeitungen, und andern dergleichen fliegenden Blättern angefüllet, so kann Pohlen gar nichts von gelehrten Monatschrifften dermalen aufweisen. ${ }^{15}$

Dies dürfte die Annahme nahelegen, dass sich Mizler de Kolof als Begründer der Warschauer Bibliothek in der Rolle eines Missionars sah, der dem unwissenden Volk eine Botschaft überbrachte und damit vollkommen neue Perspektiven eröffnete. Dies stimmt so jedoch nicht. Die Gründung der Warschauer Bibliothek ergab sich nicht aus einem einseitigen Transfer zwischen der deutschen und der polnischen Kultur, sondern war Ergebnis einer Allianz von Ideen, die dank geschickter Vermittler Gestalt annehmen konnten - hier muss neben Mizler de Kolof auch der Bischof von Kiew (Kyjiv) und Krongroßkanzler Józef Andrzej Załuski genannt werden.

Der Entstehung der Warschauer Bibliothek war das Programm einer Belebung der humanistischen Wissenschaften in Polen vorausgegangen, das Załuski 1732 in der Abhandlung Programma literarium ad bibliophilos, typothetas et bibliopegos tum et quosvis liberalium artium amatores formuliert hatte. ${ }^{16}$ Załuski gehörte zu der schmalen

12 Vgl. Hombek, Prasa i czasopisma (wie Anm. 7), S. 49 f.

13 Thomas Habel: Gelehrte Journale und Zeitungen der Aufklärung. Zur Entstehung, Entwicklung und Erschließung deutschsprachiger Rezensionszeitschriften des 18. Jahrhunderts. Bremen 2007, S. 67.

14 Vgl. Wöhlke, Mizler (wie Anm. 3), S. 86-92.

15 Warschauer Bibliothek. 1. Teil (1753), S. 9.

16 Das Programma literarium erschien zunächst 1728 in den Leipziger Neuen Zeitungen von Gelehrten Sachen. Als Einzeldruck und in erweiterter Form wurde es dann 1732 in Warschau in polnischer 
Elite der aufklärerisch orientierten Fortschrittsdenker in Polen, die sich noch vor der großen Reformwelle, die nach der Thronbesteigung Stanisław August Poniatowskis im Jahre 1764 einsetzte, für die Etablierung wissenschaftlicher, an der neuen Philosophie orientierter Institutionen in Polen engagierten. ${ }^{17}$ Laut Gerard Koziełek dürfte die Anregung zu seinem Programma literarium von Johann Burkhard Mencke, dem Herausgeber der Acta Eruditorum, ausgegangen sein, der neben Johann Christoph Gottsched und Abraham Michael Trotz zu Załuskis Bekanntenkreis in Leipzig gehörte. ${ }^{18}$ Das Programm sah eine groß angelegte bibliographische Erfassung der älteren polnischen Drucke und Manuskripte, die Edition der wichtigsten historischen Quellen sowie die Registrierung der aktuellen polnischen Bücherproduktion vor. Załuski beklagte darin ausdrücklich den Mangel an gelehrten Periodika in Polen und damit den fehlenden Informationsfluss über polnische Alt- und Neuerscheinungen in Polen und im Ausland.

Der Verwirklichung seiner Ziele dienten, neben einer Reihe von editorischen Projekten, zwei große Vorhaben: die Eröffnung der sogenannten Warschauer bzw. Załuskischen Bibliothek (Biblioteka Załuskich) in dem dazu umgebauten DaniłowiczPalast im Jahre 1747, die bis zur dritten Teilung Polens im Jahre 1795 als intellektuelles Zentrum fungierte, ${ }^{19}$ sowie die Gründung der ersten gelehrten Zeitschrift in Polens Hauptstadt, der Warschauer Bibliothek. Das erste Unternehmen gelang dank der Kooperation mit seinem Bruder, Andrzej Stanisław Załuski, Bischof von Krakau. Für das zweite war die Begegnung Józef Andrzej Załuskis mit Mizler de Kolof entscheidend, der sich bereit erklärte, das Periodikum herauszugeben und damit (wie er zumindest anfangs annahm) alleine den redaktionellen und verlegerischen Aufwand zu tragen.

Sprache und 1743 in lateinischer Übersetzung veröffentlicht. Vgl. Klimowicz, Die Frühaufklärung (wie Anm. 5), S. $163 \mathrm{f}$.

17 Vgl. ebd. Zu der Gruppe gehörte auch sein Bruder Andrzej Stefan Załuski, der mit Wolff jahrelang im Briefverkehr stand und versuchte, trotz des Widerstandes vieler Professoren die Lehren Wolffs an der Krakauer Universität durchzusetzen.

18 Vgl. Gerard Koziełek: Die deutschsprachige „Bibliothek“ in Polen. In: Ders., Reformen, Revolutionen und Reisen (wie Anm. 6), S. 40 -51, hier S. 41.

19 Die Bibliothek, deren Bestände im Jahr ihrer Eröffnung ca.18.000 Drucke und 10.000 Manuskripte, zahlreiche Landkarten, Atlanten und Kupferstiche umfassten, wurde 1794 nach der Besetzung Warschaus durch russische Truppen aufgelöst. Vgl. Elżbieta Aleksandrowska: Biblioteka Publiczna Załuskich (1747-1794) [Die öffentliche Załuski-Bibliothek (1747-1794)]. In: Dies. u.a. (Hg.): Bibliografia Literatury Polskiej. Nowy Korybut. Bd. 4: Oświecenie [Bibliographie der polnischen Literatur. Nowy Korybut. Bd. 4: Aufklärung]. Warszawa 1966, S. 99-102, hier S. 100. 


\section{Zielsetzung des Zeitschriftenprojektes}

In der Warschauer Bibliothek flossen somit Mizler des Kolofs Erfahrung und aufklärerische Einsatzbereitschaft mit Załuskis breit angelegter, auf die nationalen Bedürfnisse zugeschnittener Vision der Erneuerung polnischer Wissenschaft und Kultur zusammen. Das Periodikum stellte sowohl im Hinblick auf den Titel als auch in seiner Grundausrichtung ein Pendant zu der Załuskischen Bibliothek dar. ${ }^{20}$ Inwieweit die Zielsetzung der beiden, in der Warschauer Kulturlandschaft völlig neuen öffentlichen Institutionen übereinstimmte, lässt sich schon an dem ersten Beitrag der Warschauer Bibliothek erkennen. Es ist ein Auszug aus dem von dem Präfekten der Bibliothek Jan Daniel Janocki verfertigten Verzeichnis der in der Bibliothek vorhandenen Manuskripte (Specimen catalogi codicum manuscriptorum bibliothecae Zaluscianae), das zwar in gedruckter Form vorlag, aber nie, glaubt man den Angaben von Mizler de Kolof, im Handel erhältlich gewesen war (der Krakauer Bischof Andrzej Stanisław Załuski soll es ausschließlich an „,auswärtige Höfe und Minister, als auch an berühmte Gelehrte“ verschenkt haben). ${ }^{21}$ Was die Załuskische Bibliothek in der Sammlung und bibliographischen Erfassung von raren polnischen Drucken leistete, wurde somit durch die Warschauer Bibliothek einem breiteren Publikum kund- und gleichzeitig zumindest partiell zugänglich gemacht.

Der erste Beitrag der Warschauer Bibliothek ist aber auch symptomatisch für die redaktionelle Souveränität Mizler de Kolofs. Er erkannte zwar durchaus die Rolle seines Periodikums als einer Schwesterinstitution der Załuskischen Bibliothek an und profitierte sogar von dieser Position, gleichzeitig veröffentlichte er jedoch selten Texte kommentarlos und drückte so nahezu jedem Zeitschriftenbeitrag seinen Stempel auf.

Wir eröffnen billig unsere Warschauer Bibliothek mit dem Bücherverzeichnis von so vielen vortrefflichen Manuscripten, die in der Zaluskischen Bibliothek, oder vielmehr in der öffentlichen Warschauer Bibliothek enthalten sind. Denn diese Bibliothek ist es eben, die unsere gegenwärtige Warschauer Bibliothek erzeuget, und ohne sie würden wir gar nichts ausrichten können, da wir hingegen durch Hülfe der so ungemein zahlreichen, kostbarn und vortrefflichen Zaluskischen Bibliothek, denen Kennern der Wissenschaften so viel gutes, merkwürdiges und seltenes vorlegen werden, so sie vielleicht von Pohlen nicht vermuthet hätten. ${ }^{22}$

In den einleitenden und begleitenden Kommentaren zu dem Manuskriptenverzeichnis, aber auch in zahlreichen anderen Beiträgen unterstreicht Mizler de Kolof unermüdlich den Nutzen der öffentlichen Załuskischen Bibliothek für die Entwicklung der Wissenschaften in Polen. Mit der Warschauer Bibliothek können sich seiner Einschätzung nach auf dem deutschsprachigen Gebiet nur die Bibliotheken in Wien und

20 Vgl. hierzu auch Jarosław Kurkowski: Warszawskie czasopisma uczone doby Augusta III [Die Warschauer gelehrten Zeitschriften in der Zeit Augusts III.]. Warszawa 1994, S. 98.

21 Warschauer Bibliothek. 1. Teil (1753), S. 22.

22 Ebd., S. $13 \mathrm{f}$. 


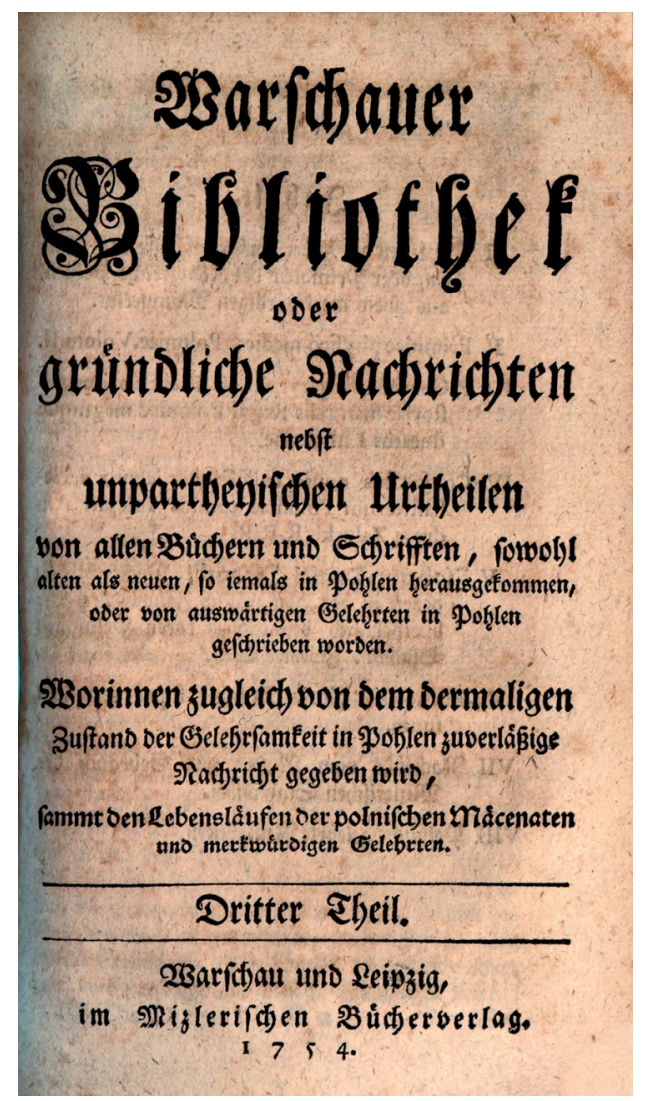

Abb. 1: Titelblatt der dritten Ausgabe der Warschauer Bibliothek von 1754 (Bayerische Staatsbibliothek München, H.lit.p. 248-1/4, S. 1, URL: http://mdz-nbn-resolving.de/urn:nbn:de:bvb:12bsb10814465-1).

in Wolfenbüttel messen. ${ }^{23}$ Immer wieder hebt er auch seine Teilhabe an den Initiativen Załuskis hervor, insbesondere die Herausgabe der Warschauer Bibliothek und deren direkten Bezug auf die reale Institution.

Die Anpassung des Inhaltes der Warschauer Bibliothek an ihre Hauptfunktion, also die Fortsetzung und Ergänzung der bio-bibliographischen und editorischen Arbeiten Józef Andrzej Załuskis, verlangte von Mizler de Kolof einen enormen Arbeitsaufwand. Bis auf die Auszüge aus den Verzeichnissen der Bibliotheksbestände ${ }^{24}$

23 Vgl. ebd., S. 22.

24 Nach dem erwähnten Manuskriptenverzeichnis im ersten Heft folgte im zweiten und dritten die umfangreiche Nachricht von denen in der Zaluskischen Bibliothek sich befindenden raren polnischen Büchern. Diese war Teil eines mehrbändigen, deutschsprachigen Verzeichnisses, das Józef Andrzej Załuski und Daniel Janocki vorbereiteten, um einzelne Bände auf der Leipziger Ostermesse zu prä- 
stammten alle in der Warschauer Bibliothek veröffentlichten Hauptbeiträge aus seiner Feder. Meist handelte es sich dabei um umfangreiche, kommentierte Paraphrasierungen der kostbarsten, oft nur handschriftlich vorhandenen Schriften aus der Załuskischen Bibliothek. ${ }^{25}$ Auch die jedes Heft abschließende Rubrik Pohlnische gelehrte Neuigkeiten wurde von Mizler de Kolof auf eigene Art und Weise gestaltet. ${ }^{26}$ Er beließ es nicht bei der bloßen Liste, deren Titelaufstellung den Korrespondenten der Bibliothek zu verdanken war, ${ }^{27}$ sondern erweiterte einzelne Einträge zu regelrechten Buchbesprechungen. Zudem wurden alle polnischen Titel der Rubrik ins Deutsche übertragen.

Aus der Vorrede zu dem ersten Heft der Warschauer Bibliothek geht hervor, dass Mizler de Kolof mit dem Transfer der Gattung, gelehrtes Journal' nach Polen und mit der entsprechenden Modifizierung dieser Gattung (im Sinne Załuskis) grundsätzlich zwei Ziele verfolgte: Einerseits hatte er vor, die polnischen Gelehrten, denen er überwiegend die Kenntnis der deutschen Sprache zutraute, ,zu mehrern Fleiß in den Wissenschaften aufzumuntern“. ${ }^{28}$ Sein Hinweis auf diejenigen polnischen Gelehrten, die kein Deutsch konnten (,den übrigen wünschen wir sie nicht $\mathrm{zu}$ lesen, weil sie verschiedenes darinnen finden möchten, so ihnen nicht anständig“), ${ }^{29}$ dürfte eine Anspielung auf die Haltung der polnischen Jesuiten gewesen sein, die sich noch um 1750 der Verbreitung der neuen Philosophie in Polen widersetzten und insbesondere die Lehren Wolffs für „ausländische Häresien“ hielten. ${ }^{30}$ Andererseits ging es de Kolof darum, den „Ausländern Gelegenheit zu geben, die Pohlnischen Scribenten besser kennen zu lernen, und sich solche zu Nutze zu machen.“"31

Es war somit ein Versuch, Polen in den Wissenstransfer zu integrieren, der sich in der Zeit der Aufklärung europaweit unter anderem dank der Entwicklung der Presse

sentieren. Insgesamt erschienen fünf Bände (Bd. 1: Dresden 1747, Bd. 2: Breslau 1749, Bde. 3-5: Breslau 1753). Vgl. Kurkowski, Warszawskie czasopisma (wie Anm. 20), S. 35.

25 Dem umfangreichen Exzerpt in der Warschauer Bibliothek folgte oft eine vollständige Edition, wie etwa im Falle des Manuskripts Historiarum Poloniae ab excessu Vladislai IV ad pacem Olivensem von Warzyniec Rudawski, das zwei Jahre nach der Zusammenfassung im ersten Heft des Periodikums von Mizler de Kolof herausgegeben wurde. Vgl. Kurkowski, Warszawskie czasopisma (wie Anm. 20), S. $209 \mathrm{f}$. 26 Sie war im gewissen Sinne eine Fortsetzung des ,Informationsbulletins‘ über polnische Neuerscheinungen, das Józef Andrzej Załuski und Daniel Janocki mindestens bis 1747 für Jacques Perard, den Redakteur der Zeitschrift Nouvelle Bibliotheque Germanique, vorbereiteten. Vgl. Heinz Lemke: Die Brüder Załuski und ihre Beziehungen zu Gelehrten in Deutschland und Danzig. Berlin 1958, S. $119-134$.

27 Laut Jarosław Kurkowski, der den Briefwechsel der Brüder Załuski analysiert hat, gab es in den Jahren 1747-1763 mindestens 14 an verschiedenen Orten in Polen lebende Personen, die Informationen über Neuerscheinungen nach Warschau schickten. Vgl. Kurkowski, Warszawskie czasopisma (wie Anm. 20), S. $111 \mathrm{f}$.

28 Warschauer Bibliothek. 1. Teil (1753), S. 10.

29 Ebd., S. 12.

30 Vgl. Lemke, Die Brüder Załuski (wie Anm. 26), S. 169.

31 Warschauer Bibliothek. 1. Teil (1753), S. 10. 
intensivierte. Dem Deutschen als einer „in Europa so gangbare[n] Sprache“ ${ }^{\text {32 }}$ schrieb de Kolof dabei den Status einer Brücke zu, die diesen Transfer erst möglich machte. Die Wahl der deutschen und nicht etwa der lateinischen Sprache für die Warschauer Bibliothek korrespondierte auch mit den Aktivitäten Józef Andrzej Załuskis, der ja enge Kontakte zu deutschen Gelehrten und Verlegern unterhielt und auch seine für die Leipziger Ostermesse und für deutsche Zeitschriften bestimmten Bücherverzeichnisse auf Deutsch drucken ließ. Nicht ohne Bedeutung mag dabei auch die Orientierung an der in Deutschland populären Gattung des ,gelehrtes Journals‘ gewesen sein, das um die Mitte des 18. Jahrhunderts nur noch selten auf Latein erschien. Dass die Fortsetzung der Warschauer Bibliothek (ab 1756 als Acta Litteraria) doch auf Latein erfolgte, war wiederum auf den ausdrücklichen Wunsch des neuen Mäzens Fürst Jablonowski zurückzuführen, der kein Deutsch konnte. ${ }^{33}$

\section{Zur Rezeption der Warschauer Bibliothek und ihren soziokulturellen Hintergründen}

Ganz im Sinne Mizler de Kolofs wurde die Warschauer Bibliothek im deutschsprachigen Ausland nicht nur bemerkt, sondern auch enthusiastisch aufgenommen. In zahlreichen Rezensionen unterstrich man ihren Pioniercharakter. ${ }^{34}$ Laut den Leipziger Neuen Zeitungen von gelehrten Sachen, die jedes Heft des Periodikums einzeln besprachen, wurde mit der Gründung der Warschauer Bibliothek die Chance zu einem Durchbruch in der Entwicklung der Gelehrsamkeit in Polen geschaffen:

\footnotetext{
Dieses ist die erste Monathschrift, welche uns Pohlen geliefert, und wir wünschen, daß die übrigen Gelehrten dieses Landes, durch ein so gutes Beyspiel mögen angereizet werden, uns gleichfalls die bey ihnen noch verborgenen Schätze, bekannt zu machen; wir können es auch, bey unsern aufgeklärten Zeiten, mit Grunde hoffen. ${ }^{35}$
}

Doch entgegen dieser Prognose fand die Warschauer Bibliothek in Polen selbst nicht die erhoffte Resonanz. Mizler de Kolofs in der Vorrede vorgebrachter Vorsatz, es nicht dahin kommen zu lassen, dass seine Schrift „zum Kampfplatz der Gelehrten in Pohlen“ werde, ${ }^{36}$ erwies sich als vollkommen unnötig. Während der ganzen Erscheinungsdauer der Warschauer Bibliothek hat er weder gelegentliche Beiträger noch

32 Ebd., S. 12.

33 Vgl. Kurkowski, Warszawskie czasopisma uczone (wie Anm. 20), S. 59 f.

34 Vgl. Göttingische Anzeigen von gelehrten Sachen 129 (1756), S. 1170 - 1173; Freymüthige Nachrichten von neuen Büchern und andern zur Gelehrtheit gehörigen Sachen 13 (1756), S. 39f.; Freye Urtheile und Nachrichten zum Aufnehmen der Wissenschaften und Historie überhaupt 12/58 (1755), S. 464.

35 Neue Zeitungen von gelehrten Sachen 39 (1754), S. 346 -348, hier S. 348.

36 Warschauer Bibliothek. 1. Teil (1753), S. 11. 
langfristige Mitarbeiter gewinnen können, musste daher das Material durchgängig selbst erstellen. ${ }^{37}$ Somit hat er am eigenen Leibe die Bestätigung der von ihm eigens in der Vorrede zur ersten Nummer formulierten Diagnose des polnischen Pressemarktes gespürt:

Es ist dieses gar nicht die Ursach, als wenn es denen Pohlen an Stoff hierzu [zu der Gründung von Periodika] fehlte, sondern es fehlt nur an Personen, die diese Mühe über sich nehmen. Die Ernde ist groß, aber es sind wenig Arbeiter. ${ }^{38}$

Bedenkt man die Ständestruktur der polnischen Gesellschaft im 18. Jahrhundert, so war es in Polen kaum möglich, dem hier von Mizler de Kolof identifizierten Mangel an „Arbeitern“ entgegenzuwirken. Mit ihnen meinte er Gelehrte, Verleger, Drucker, mit anderen Worten: Vertreter des gebildeten Bürgertums, die in anderen Ländern Europas die eigentliche soziale Trägerschicht der Aufklärung ausmachten. In Polen war hingegen das Bürgertum nicht nur nicht zahlreich, ${ }^{39}$ sondern auch wirtschaftlich und sozial schwach. Zudem stand es unter starkem Einfluss der Lebensweise und der Wertvorstellungen des Adels, der die polnische Politik, Wirtschaft und Kultur im 18. Jahrhundert und weit darüber hinaus dominierte. ${ }^{40}$ Die Aufklärung in Polen konnte daher, zumindest hinsichtlich ihrer Träger und der ,kulturellen Infrastruktur, nicht bürgerlich orientiert sein. Andererseits bildeten auch die fortschrittlich denkenden Adligen eine nur schmale, elitäre Gruppe. ${ }^{41}$

Der Mangel an „Arbeitern“ galt auch für die Załuskische Bibliothek, die, wie de Kolof in der Warschauer Bibliothek berichtete, trotz ihrer hervorragenden Bestände von sehr wenigen Gelehrten besucht wurde. ${ }^{42}$ Załuski hatte zudem mit der nachläs-

37 Auf diese Erfahrung ging de Kolof im ersten Heft der Acta Litteraria ein, wo er feststellte, dass ein Mensch es nicht schaffen könne, allein ein gelehrtes Periodikum herauszugeben, und es daher in Zusammenarbeit geschehen solle, zu der er die Gelehrten in Polen ausdrücklich aufforderte. Vgl. Acta Litteraria. 1. Teil (1756), S. 10.

38 Warschauer Bibliothek. 1. Teil (1753), S. 9.

39 Der Anteil des Bürgertums an der gesamten Bevölkerung in Polen betrug selbst am Ausgang des 18. Jahrhunderts ca. sechs Prozent. Der Bevölkerungsanteil des polnischen Adels lag hingegen, je nach Region, bei acht bis fünfzehn Prozent. Vgl. Marcin Cieński: Formacja oświeceniowa w literaturze Polski i Niemiec [Die Formation der Aufklärung in der Literatur Polens und Deutschlands]. Wrocław 1992, S. 40 f.; Eduard Mühle: Genese und frühe Entwicklung des Adels in polnischer Sicht. In: Ders. (Hg.): Studien zum Adel im mittelalterlichen Polen. Wiesbaden 2012, S. 1-12, hier S. $1 \mathrm{f}$.

40 Vgl. ebd.

41 Die Aufklärung in Polen setzte relativ spät ein (die Hochphase ab 1764) und umfasste vorrangig eine schmale adlige, katholische Elite. Die katholische Kirche hat einerseits die konservativen, reformfeindlichen Haltungen unterstützt. Andererseits gehörten viele der Hauptakteure der neuen Bewegung (Stanisław Konarski, Ignacy Krasicki, Franciszek Bohomolec oder die Brüder Załuski) dem Klerus an. Die polnische Aufklärung war auch stark durch die andauernde politische Krise determiniert, die in den Teilungen kulminierte. Vgl. Cieński, Formacja oświeceniowa (wie Anm. 39), S. 42-52. 42 Die niedrigen Besucherzahlen der Załuskischen Bibliothek werden in vielen zeitgenössischen Überlieferungen bestätigt. Vgl. Jan Kozłowski: Szkice o dziejach biblioteki Załuskich [Skizzen über die Geschichte der Załuski-Bibliothek]. Wrocław u.a. 1986, S. 40 - 45. 
sigen Reaktion auf seine im Kurier Polski veröffentlichten Bitte um die Zusendung von ,Pflichtexemplaren' oder zumindest von Informationen über Neuerscheinungen an die Bibliothek zu kämpfen. ${ }^{43}$ Laut de Kolof war die Stiftung der Załuskischen Bibliothek ihrer Zeit voraus - und dies traf wohl auch für die Warschauer Bibliothek zu. Nach seiner Einschätzung würden erst die künftigen Generationen die Bedeutung dieser Institution richtig einschätzen und sie „noch mehr preisen [...] als die ietztlebenden, wovon nur einige wenige sich dieses herrlichen Bücherschatzes $\mathrm{zu}$ bedienen wissen." ${ }^{\text {44 }}$

Dass man in Polen lange Zeit an gelehrten (periodischen und nicht-periodischen) Druckerzeugnissen wenig interessiert war, hing weitgehend damit zusammen, dass sich der polnische Adel generell nur sehr langsam an die gedruckten Formen der Informationsvermittlung gewöhnte. Noch um die Mitte des 18. Jahrhunderts wurden in Polen alte Formen der Wissensübermittlung wie orale Kommunikation sowie handgeschriebene (eher konservative Denkschemata verbreitende) Zeitungen und Flugblätter bevorzugt. ${ }^{45}$ Der Informationsaustausch erfolgte in erster Linie nicht in den Städten, die ökonomisch und kulturell schwach waren (selbst die Hauptstadt Warschau zählte um 1764 nur 23.000 Einwohner), ${ }^{46}$ sondern auf einflussreichen Magnatenhöfen.

So blieb auch die Zahl der potenziellen Leser eines gelehrten Journals wie der Warschauer Bibliothek stark beschränkt. Da infolgedessen auch die Möglichkeit ihrer Selbstfinanzierung kaum in Aussicht stand, war Mizler de Kolof von Anfang an auf das Mäzenatentum angewiesen, das sich in Polen, folgt man seiner Einschätzung, erst langsam zu entwickeln begann. Er vermisste Mäzene, „welche die Gelehrten in Pohlen nicht nur mit Höflichkeiten und Lobeserhebungen, sondern würklichen Wohltaten aufmuntern“". ${ }^{47}$ Eine Ausnahme in dieser Hinsicht war in seinen Augen Józef Andrzej Załuski, „unser polnischer Mäcenas“, wie er ihn in der Warschauer Bibliothek nannte. ${ }^{48}$

43 Vgl. Hombek, Prasa i czasopisma (wie Anm. 7), S. 54-56.

44 Warschauer Bibliothek. 1. Teil (1753), S. 17.

45 Vgl. Kazimierz Maliszewski: Komunikacja społeczna w kulturze staropolskiej: studia z dziejów kształtowania się form i treści społecznego przekazu w Rzeczypospolitej Szlacheckiej [Gesellschaftliche Kommunikation in der altpolnischen Kultur. Studien zur Entstehungsgeschichte der Formen und Inhalte gesellschaftlicher Überlieferungen in der Adelsrepublik]. Toruń 2001, S. 36-39.

46 Vgl. Mieczysław Klimowicz: Oświecenie [Aufklärung]. Warszawa 2006, S. 12.

47 Warschauer Bibliothek. 2. Teil (1754), S. 149.

48 Ebd., S. 123. 


\section{Mizler de Kolof in der Kritik}

Das anfänglich gute Einvernehmen Mizler de Kolofs mit den Brüdern Załuski geriet im Laufe der Herausgabe der Warschauer Bibliothek ins Wanken. Als entscheidend erwies sich dabei Mizler de Kolofs redaktionelle Autonomie, die sich vorrangig in der Freiheit und Strenge seiner auf die polnischen Verhältnisse bezogenen Urteile manifestierte. Damit verspielte er die Aussichten auf eine langfristige finanzielle Unterstützung des Periodikums.

Auch wenn Mizler de Kolof den Großteil seiner Beiträge in der Warschauer Bibliothek, gemäß der Zielsetzung Józef Andrzej Załuskis, der Geschichte des polnischen Schrifttums widmete, so war sein Blick stets auch nach vorne, auf die neuesten Debatten und Probleme gerichtet. Die in der Warschauer Bibliothek veröffentlichten Texte und historiographischen Arbeiten versah er daher immer wieder mit Kommentaren über den aktuellen Zustand der Wissenschaft in Polen, in denen sich Fortschrittsoptimismus und Aufbruchstimmung mit kritischen Stellungnahmen verbanden. Nach über zehn in Polen verbrachten Jahren trat Mizler de Kolof sowohl als Förderer als auch als Kritiker seiner Wahlheimat auf. In seinen Ausführungen integrierte er die Sicht eines fast Einheimischen, der über einen tiefen Einblick in die polnischen Angelegenheiten verfügte, mit der Außenperspektive eines aufgeklärten, rational denkenden Vertreters des deutschen Bürgertums.

Folgt man dem Blickwinkel Mizler der Kolofs, so lassen sich in Polen um die Mitte des 18. Jahrhunderts Anfänge von Prozessen und Reformen beobachten, die davon zeugen, dass die Zirkulation der aufklärerischen Ideen das Land erreicht hatte. Seinen Berichten zufolge begann man in Polen, Mathematik, Experimentalphilosophie und Physik zu unterrichten. Ebenso fing man an - das Verb ,anfangen' ist symptomatisch für seine Sicht der Dinge -, Wert auf die Reinheit der lateinischen Sprache und der Nationalsprache zu legen. Auch versuchte man die polnische Geschichtsschreibung $\mathrm{zu}$ kultivieren und gelehrte Korrespondenzen zu pflegen, das polnische Theater zu verbessern, fremde Literatur ins Polnische zu übersetzen und in Polen aufzuführen. De Kolof exponierte dabei gerne seine eigene Rolle, indem er etwa den unentgeltlichen Griechischunterricht erwähnte, den er sechs piaristischen Priestern „aus Liebe gegen die pohlnischen Gelehrten“ erteilte. ${ }^{49}$ Kurzum, in seinen Beiträgen für die Warschauer Bibliothek war er bemüht, den Glauben an die Möglichkeit eines schnellen Fortschritts in Polen zu vermitteln: ,wenn man in Pohlen so fortfähret, wie man angefangen, so ist gar kein Zweifel, daß nicht mit der Zeit es ebensoviel zum Wachsthum der Wissenschaften, als andere Länder, beytragen sollte. “50

Doch die anerkennende Hervorhebung der neuen, erst im Ansatz vorhandenen ,Bildungspraktiken“ implizierte bei de Kolof auch Bezugnahmen auf Probleme und Defizite, die bei weitem noch nicht aufgehoben waren und der Durchsetzung neuer

49 Warschauer Bibliothek. 1. Teil (1753), S. 80.

50 Ebd. 
Ideen weiterhin im Wege standen. Es geht in erster Linie um das anhaltende Desinteresse der Polen an der Entwicklung der Wissenschaften und die daraus resultierende Rückständigkeit des Landes:

Ja es scheinet bey einigen einen Anstoß zu verursachen, wenn man sich für andern in den Wissenschaften hervorthut. Viele sehen den großen Nutzen der Wissenschaften im Staat, im Krieg und Frieden, im Handel und Wandel und der Landwirthschafft noch nicht recht ein, und glauben, daß ein mit alten Weinen angefüllter Keller nützlicher denn die schönste Bibliothek sey. ${ }^{51}$

Die Anspielung auf den Weinkeller als Gegenstand des adligen Stolzes, mit der Mizler de Kolof hier einen Zusammenhang zwischen der Rückständigkeit Polens und dem Lebensstil des polnischen Adels herstellte, durfte durchaus als beleidigend aufgenommen werden. Mizler de Kolof konnte sich jedoch in seinen Urteilen über den polnischen Adel noch weit kompromissloser zeigen, indem er etwa die von Generation zu Generation gepflegte übermäßige Anhänglichkeit der polnischen Adligen an ihrem „Schlachectwo“ (adligen Stand) anprangerte, und zwar als eine Beschaffenheit, die andere Lebenssphären, insbesondere Bildung und Erziehung, negativ determiniere und demzufolge Ursache des zögerlichen Fortschritts sei:

Fraget man nach der Ursach, so sind wir mit der richtigen Antwort gleich fertig: von der nachläsigen Erziehung junger Leute; welche daher kommt, daß Academien und Schulen nicht so bestellt sind, wie sie seyn sollten und könnten. Der häutige Adel ist mehr von seiner Schlachectwo als einem wahren Trieb was gründliches zu lernen eingenommen. ${ }^{52}$

Die Reaktionen auf Urteile dieser Art ließen nicht lange auf sich warten. Andrzej Stanisław Załuski, der Bischof von Krakau, der die Warschauer Bibliothek langfristig finanzieren sollte (sein Bruder Józef Andrzej Załuski konnte lediglich die Kosten der ersten Hefte der Zeitschrift bestreiten), versagte seine Unterstützung. Tief gekränkt äußerte er sich in den Briefen an seinen Bruder abschätzig über Mizler de Kolof und dessen Arbeit. Dem Herausgeber der Warschauer Bibliothek warf er vor, dass er in seinem „Büchlein“ die polnische Nation verleumde, obwohl er vom polnischen Brot lebe. ${ }^{53}$ Hinzu kam noch, dass Mizler de Kolof in jedem Heft der Warschauer Bibliothek einen Beitrag über von ihm selbst erfundene und hergestellte Arzneimittel unterbrachte (im ersten Heft handelte es sich um einen Text über das „Mizlerische Wundbalsam“ ${ }^{\text {54 }}$ - formal gesehen eine Zusammenfassung der von Mizler de Kolof 1747 in Erfurt verteidigten Dissertation). Auch das wurde ihm von Andrzej Stanisław Załuski zur Last gelegt. ${ }^{55}$

51 Ebd., S. 22f.

52 Warschauer Bibliothek. 2. Teil (1754), S. 124.

53 Vgl. Lemke, Die Brüder Załuski (wie Anm. 26), S. 141.

54 Vgl. Warschauer Bibliothek. 1. Teil (1753), S. 75-79.

55 Vgl. Lemke, Die Brüder Załuski (wie Anm. 26), S. 141. 
Nachdem Józef Andrzej Załuski ihn mit der Kritik konfrontiert und ihm die Briefe des Bruders gezeigt hatte, unternahm Mizler de Kolof noch einen Versuch, den Krakauer Bischof umzustimmen. Doch weder seine Erklärung, er habe mit der Herausgabe der Warschauer Bibliothek ,zum Ruhm der polnischen Nation und der Załuskischen Bibliothek ${ }^{* 56}$ gewirkt, noch die Versicherung, dass in dem kommenden (dritten) Heft die Einwände des Bischofs größtenteils bereits berücksichtigt worden seien, konnten etwas an der Sache ändern. Andrzej Stanisław Załuski war zudem nicht der einzige, der negative Urteile über die Warschauer Bibliothek verbreitete. Aus einem Schreiben Jan Daniel Janockis, dem Präfekten der Załuskischen Bibliothek, an den Ermländischen Bischof Adam Stanisław Grabowski geht hervor, dass im Jahre 1756 ein handschriftliches Flugblatt über die Tätigkeit Mizler de Kolofs im Umlauf war, in dem er als ein „falscher Kritiker der polnischen Nation“ bezeichnet wurde. ${ }^{57}$

Schließlich wurde Mizler de Kolof, wie er selbst in der Vorrede zu der gebundenen Ausgabe aller vier Bände der Warschauer Bibliothek zugab, „für Pohlen zu arbeiten aufzuhören genöthiget““ ${ }^{58}$ Die Kritik an den lokalen Missständen, die in seinen Augen eine legitime Praxis war, wurde in der polnischen Adelsrepublik als ein Normverstoß angesehen. Damit konnte er sich, trotz seiner ansonsten weitgehenden Anpassungsbereitschaft an die polnischen Bedürfnisse und Eigentümlichkeiten (etwa bei der Gestaltung der Warschauer Bibliothek) kaum abfinden. Noch in der Warschauer Bibliothek plädierte er für die Legitimität von Kritik im öffentlichen Diskurs in Polen:

Gar viele können in Pohlen die Wahrheit durchaus nicht vertragen, und ohngeachtet die Freyheit in Polen herrschen soll, so will man doch die redlichsten Scribenten zu Sclaven machen. [...] Wir haben es selber bey der Herausgebung dieser Schrift erfahren, dass die geringste Critik, wenn man gleich von der Wahrheit derselben überzeigt ist, anstößig ausfällt. Im Reich der Wissenschaften gehet die Wahrheit über alles [...]. Es ist der polnischen Nation keine Schande, wenn man die Mißbräuche und Laster in Pohlen überhaupt tadelt. ${ }^{59}$

Doch die öffentliche Sphäre funktionierte um die Mitte des 18. Jahrhunderts in Polen anders als etwa in Deutschland, wo sich im Zuge der Aufklärung und der mit ihr einhergehenden Emanzipation des Bürgertums ein, um es mit Günther Lottes zu sagen, „literarisch-publizistischer Markt und ein Publikum als Lese- und Urteilsgemeinschaft etablierten“. ${ }^{60}$ Heinz Lemke verweist darauf, dass die negativen Urteile, die Mizler de Kolof etwa über den schlechten Zustand des polnischen Bildungswesens in der Warschauer Bibliothek erhob, im Grunde genommen weitgehend den Ansichten des Bischofs von Krakau entsprachen, der sich ja eifrig für die Reform der Krakauer

56 Zit. nach ebd., S. 142.

57 Zit. nach Kurkowski, Warszawskie czasopisma (wie Anm. 20), S. 225.

58 Warschauer Bibliothek. 4. Teil (1754), S. 254.

59 Warschauer Bibliothek. 3. Teil (1754), S. $251 \mathrm{f}$.

60 Günter Lottes: Strukturtypen und Strukturregionen des Kulturtransfers. Auf der Suche nach der Kulturgesamtheit im Europa der Kulturvielfalt. In: Agnieszka Pufelska, Iwan-Michelangelo D’Aprile (Hg.): Aufklärung und Kulturtransfer in Mittel- und Osteuropa. Hannover 2009, S. 13-34, hier S. 16. 
Universität einsetzte. Was jedoch in der Abgeschiedenheit der Kabinette und Paläste zulässig war, durfte nicht zum Gegenstand kritischen öffentlichen Räsonnements werden. Schwer wog auch, dass die Kritik von einem Ausländer kam, dazu noch von einem Vertreter der in der polnischen Adelsrepublik um die Mitte des 18. Jahrhunderts wenig beachteten Schicht des Bürgertums. ${ }^{61}$

Bezeichnenderweise hatte Mizler de Kolof in Polen keine Chance, den Konflikt, den seine kritischen Kommentare in der Warschauer Bibliothek herbeiführten, öffentlich auszutragen. Es meldete sich weder ein Opponent, der bereit gewesen wäre, auf seine Anstoß erregenden Urteile offen einzugehen, noch gab es (sieht man von der Warschauer Bibliothek selbst einmal ab) ein Medium, wo die Polemik hätte stattfinden können. Die einzige gedruckte Spur der Kritik an der Warschauer Bibliothek fand sich im Lexicon derer itztlebenden Gelehrten in Polen von Jan Daniel Janocki:

\footnotetext{
Der Herr Hofrath und Hofmedicus Mizler besitzet eine große Munterkeit, und redet und schreibet auch gern die Wahrheit, bezeiget sich aber, in seinen Beurteilungen derer Polen, allzustreng, und misset der gesammten, zu großen und rühmlichen Unternehmungen, von Natur angetriebenen Nation, solche Fehler bey, die doch nur, wie in andern wohlgesitteten, und aufs weislichste eingerichteten Republiken, von einzelnen niederträchtigen Personen begangen werden. Er ist auch für seine Medicinische Ausarbeitungen, und Chymische Erfindungen, so sehr eingenommen, daß er solche, zum größten Verdrusse derer Liebhaber Polnischer Sachen, überall zu häufig einschaltet. ${ }^{62}$
}

Der kritische Eintrag Janockis deckt sich größtenteils mit den Einwänden Andrzej Stanisław Załuskis gegenüber der Warschauer Bibliothek, auch im Hinblick auf die als aufdringlich empfundene Werbung Mizler de Kolofs für seine medizinischen Erzeugnisse. Was Janocki hier jedoch auf den Punkt bringt und bei der Entrüstung über Mizler'sche Urteile die entscheidende Rolle gespielt haben dürfte, ist die Überzeugung von der Besonderheit der polnischen, „zu großen und rühmlichen Unternehmungen“ bestimmten Nation (wobei mit ,Nation` in Polen zu dieser Zeit nur der Adel gemeint war), die als solche grundsätzlich keiner Kritik unterzogen werden durfte. Dieses in Polen im 18. Jahrhundert gängige Denkmuster war eines der konstitutiven Elemente des Sarmatismus, der die Selbstwahrnehmung und das Zusammengehörigkeitsgefühl breiter Teile der polnischen Szlachta ab dem 16. bis zum Ende des 18. Jahrhunderts prägte.

\section{Sarmatismus und Aufklärung}

Der Begriff des Sarmatismus ist auf den Mythos der Abstammung des polnischen Adels von dem antiken Geschlecht der Sarmaten zurückzuführen. Aus dem Mythos entwickelte sich eine geistige Haltung, in dessen Zentrum Traditionsbewusstsein,

61 Vgl. Lemke, Die Brüder Załuski (wie Anm. 26), S. 141.

62 Jan Daniel Janocki: Lexicon derer itztlebenden Gelehrten in Polen. Erster Teil. Breslau 1755, S. 109. 
Katholizismus und der Stolz des polnischen Adels auf seine, goldene Freiheit', die ihm einen außerordentlichen Status im Staat gewährte, standen. ${ }^{63}$ In diesem Kontext rüttelten Mizler de Kolofs harte Urteile über die polnischen Adligen an den Grundpfeilern des polnischen Nationalbewusstseins. Sie waren auch im gewissen Sinne ihrer Zeit voraus, denn die Kritik am Sarmatismus wurde in Polen erst um die Mitte der 1760er Jahre möglich, vorwiegend unter dem Einfluss der um König Stanisław August Poniatowski und die Zeitschrift Monitor gruppierten aufgeklärten Autoren (unter ihnen auch Mizler de Kolof), die den Sarmatismus für die Rückständigkeit, Isoliertheit und Megalomanie des Adels verantwortlich machten. ${ }^{64}$

Der Bezug auf den Sarmatismus stellt auch den Kulturtransfer, der mit der Warschauer Bibliothek möglich wurde, in ein neues Licht. Folgt man der Perspektive von Stanisław Roszak, so lagen den Modernisierungstendenzen in Polen bis in die Mitte des 18. Jahrhunderts keine aufklärerischen Ideen, sondern sarmatische Ideale zugrunde. Die allgemein postulierte Wiederbelebung des polnischen Staates sollte demnach nicht infolge der Adaptierung von fremden (aufklärerischen) Mustern erfolgen. Vielmehr wurde die Rückwendung zur Tradition und Vergangenheit, zu den alten Glanzzeiten der polnischen Adelsrepublik postuliert. ${ }^{65}$ Daraus resultierte die auch in der Konzeption der Warschauer Bibliothek sichtbare Tendenz zur Aufwertung der literarischen nationalen Errungenschaften vergangener Zeiten. Nach Roszak würde es schwerfallen, Mizler de Kolof für einen bewussten Befürworter der sarmatischen Ideologie zu halten. Doch auch er hat, bedenkt man die Publikationen polnischer Schriften aus dem 16. und 17. Jahrhundert in der Warschauer Bibliothek (hierzu könnten auch die zahlreichen Neueditionen älterer polnischer Literatur in Mizler de Kolofs Druckerei gezählt werden) seinen Anteil an der Kultivierung der Vergangenheit und Aktualisierung der alten sarmatischen Tugenden. ${ }^{66}$

Die Frage nach dem Verhältnis zwischen Sarmatismus und Aufklärung, insbesondere danach, ob es legitim ist, von einem ,aufgeklärten Sarmatimus‘ zu sprechen, ist vielfach diskutiert worden. ${ }^{67}$ Geht man, Martin Fabers Vorschlag folgend, von dem ursprünglichen Gebrauch des Begriffs ,Sarmatismus‘ aus, den aufgeklärte Reformer aufgebracht hatten, um damit eine Haltung zu definieren, an der sie Kritik üben wollten, so müssen Aufklärung und Sarmatismus als separate Phänomene oder sogar Gegenströmungen definiert werden. ${ }^{68}$ Auch im Sinne Stanisław Roszaks stellen sie im

63 Vgl. Martin Faber: Das Streben des polnischen Adels nach dem Erhalt seiner Privilegien. Zur ursprünglichen Bedeutung des Wortes „Sarmatismus“. In: Jahrbücher für Geschichte Osteuropas NF 57/3 (2009), S. 374-401, hier S. 378-384.

64 Vgl. ebd., S. 379.

65 Vgl. Stanisław Roszak: Środowisko intelektualne i artystyczne Warszawy w połowie XVIII wieku. Między kulturą sarmatyzmu i oświecenia [Das intellektuelle und künstlerische Umfeld Warschaus in der Mitte des 18. Jahrhunderts. Zwischen sarmatischer Kultur und Aufklärung]. Toruń 1997, S. 23 - 26. 66 Vgl. ebd., S. 131.

67 Vgl. Faber, Das Streben des polnischen Adels (wie Anm. 63), S. 374-377.

68 Vgl. ebd., S. 398. 
18. Jahrhundert nebeneinander vorkommende Erscheinungen dar, wobei es der Sarmatismus war, der bis in die 1760er Jahre die Dynamisierung des literarischen und wissenschaftlichen Lebens in Polen vorantrieb. ${ }^{69}$

In der Warschauer Bibliothek kam es, wenn auch nur für kurze Zeit, zu einer einzigartigen Koexistenz von sarmatischen und aufklärerischen Elementen. Was im Sinne Józef Andrzej Załuskis der Glorifizierung der nationalen Vergangenheit und der angesehenen Magnatengeschlechter diente, war für Mizler de Kolof ein Prätext für eine Art Bildungskampagne. Deren Hauptgegenstände waren vorrangig, wie bereits aufgezeigt, die aufzuhebende Rückständigkeit des Adels und die Reform des Erziehungswesens. De Kolof plädierte aber auch für einen kritischen, selektiven Umgang mit historischen Quellen, insbesondere für die Ablehnung von Legenden und mündlichen Überlieferungen, die selbst bei von ihm geschätzten Autoren vorkamen (wie etwa bei Jan Wawrzyniec Rudawski, dessen Schrift Historiarum Poloniae er zunächst in die Warschauer Bibliothek aufnahm und dann in seinem Verlag herausgab): „Es ist eine Schande, wenn in einem sonst guten Geschichtsschreiber [...] Mährchen anzutreffen" sind..$^{70}$ In Polen, wo man das Bedürfnis nach einer kritischen Untersuchung der historischen Quellen erst in der zweiten Hälfte des 18. Jahrhunderts zu diskutieren begann, gehörten seine Kommentare zu den ersten, die auf dieses Problem eingingen. ${ }^{71}$

\section{Mizler de Kolofs weitere Aufklärungsanstrengungen}

Nachdem Mizler de Kolof die weitere Herausgabe der Warschauer Bibliothek verwehrt worden war, entschied er sich für die Gründung neuer Periodika. Dies zeugt von der Rolle, die er der Presse als einem Instrument der Aufklärung und Wissensvermittlung zumaß, sowie möglicherweise auch von der Hoffnung auf persönlichen Erfolg und einen, für den Vertreter des Bürgertums äußerst schwierigen Aufstieg in der polnischen Adelsrepublik. Dieses Mal versuchte er jedoch die Perspektive des ,Apostels ‘ mit derjenigen eines lokalen Akteurs zu verbinden, der sich in einem viel stärkeren Maße als bisher an die polnischen Bedingungen anpasste. Die Fortsetzung des Transferprozesses, den er mit der Warschauer Bibliothek in Gang gebracht hatte, scheint nur in einem beschränkten Rahmen und unter Ausschluss kritischen Räsonnements möglich gewesen zu sein.

So war sein nächstes Periodikum, die Acta Litteraria (herausgegeben in Latein unter dem Mäzenat des Fürsten Józef Aleksander Jabłonowski), zwar in vielfacher Hinsicht eine Fortsetzung der Warschauer Bibliothek. Er benutzte weiterhin, dank der fortdauernden Zusammenarbeit mit Józef Andrzej Załuski, die Bestände und den

69 Vgl. Roszak, Środowisko intelektualne i artystyczne Warszawy (wie Anm. 65), S. 150 f.

70 Warschauer Bibliothek. 1. Teil (1753), S. 74.

71 Vgl. Kurkowski, Warszawskie czasopisma (wie Anm. 20), S. 170. 
Informationsdienst der Załuskischen Bibliothek. Doch vermied de Kolof darin nun direkte Kritik an den Auswüchsen der Adelsrepublik. ${ }^{72}$ Kein Platz für seine Urteile über die polnische Szlachta findet sich auch in seinen 1758 gegründeten Nowe wiadomości ekonomiczne i uczone [Neue ökonomische und gelehrte Nachrichten], dem ersten populärwissenschaftlichen Periodikum in Polen. ${ }^{73}$ Dasselbe gilt auch für den Patriota Polski kartki tygodniowe zawierający [Der polnische Patriot wöchentliche Blätter enthaltend] von Tobias Bauch, die erste moralische Wochenschrift in polnischer Sprache, die Mizler de Kolof mitfinanzierte und herausgab. ${ }^{74}$

Eine Wende erfolgte dagegen mit dem Warschauer Monitor (1765-1785), dem wichtigsten Presseorgan der polnischen Aufklärung, der ganz neue Perspektiven für den öffentlichen kritischen Diskurs in Polen eröffnete. ${ }^{75}$ Die radikalste Kritik, auch am Sarmatismus, kam im Monitor aus der Feder des Bischofs Ignacy Krasicki. Doch auch Mizler de Kolof, der anfänglich nur als Verleger und in den 1770er Jahren zunehmend auch als Autor von Beiträgen auftrat, ${ }^{76}$ konnte sich nun (neben zahlreichen Vorschlägen zu einer umfassenden Staatsreform) schärfere Stellungnahmen gegenüber der polnischen Gesellschaft erlauben und damit letztendlich seine in der Warschauer Bibliothek vertretene These bestätigen, dass es ,der polnischen Nation keine Schande [ist], wenn man die Mißbräuche und Laster in Pohlen überhaupt tadelt.“77

72 Vgl. Klimowicz, Polsko-niemieckie pogranicza literackie (wie Anm. 2), S. 30.

73 Vgl. Ewa Wójcik, Grażyna Wrona, Renata Zając: Polskie czasopisma popularno-naukowe do 1939 roku. Dzieje i rozwój [Polnische populärwissenschaftliche Zeitschriften bis 1939. Geschichte und Entwicklung]. Kraków 2018, S. $83-87$.

74 Vgl. Hombek, Prasa i czasopisma (wie Anm. 7), S. 72.

75 Anders als die meisten anderen europäischen Vertreter des als genuin bürgerlich geltenden Pressegenres der ,moralische Wochenschrift‘ ging der polnische Monitor aus der Initiative des Königs hervor und war für das adlige Publikum vorgesehen. Mizler de Kolof war (bis zu seiner Nobilitierung im Jahre 1768) einer der wenigen Mitarbeiter bürgerlicher Herkunft in dem elitären Kreis seiner Herausgeber.

76 Allein im Jahr 1775 stammten 59 Nummern des Monitor von ihm. Vgl. Aleksandrowska, Monitor (wie Anm. 9), S. LXXXIX.

77 Warschauer Bibliothek. 3. Teil (1754), S. 252. 
